\title{
Interactive comment on "The influence of wind and land evapotranspiration on the variability of moisture sources and precipitation of the Yangtze River Valley" by Astrid Fremme and Harald Sodemann
}

\section{Anonymous Referee \#1}

Received and published: 11 March 2019

\section{General comments:}

The moisture source for precipitation over the lower Yangtze River Valley has been studied in this manuscript. The study is conducted using trajectories calculated from a Lagrangain particle dispersion model "FLEXPART V8.2" and a Lagrangian moisture source diagnostic "WaterSip". This is an important topic with profound socio-economic values and has been widely studied previously. Authors of this manuscript make effort to compare findings herein with previous studies and explore new diagnoses to further evaluate contributions from land and ocean, i.e., the second-order moisture source

Printer-friendly version

Discussion paper 
from land. Although, it is just briefly mentioned in the manuscript, the idea of finding a common measure to compare different results from different studies is encouraging. I would like suggest to accept this manuscript if authors sufficiently answer following questions.

Specific comments:

$>$ Page 5, Lines 24-26 and Page 11, Lines 4-6: information inconsistency. In former, I have learned that $95 \%$ of moisture source is attributable by WaterSip to precipitation. However, in latter, it says, I quote, "The WaterSip summer precipitation deviations ... with an average of $-20.5 \%$. This is a typical bias for Lagrangian diagnostics (Sodemann et al., 2008)." Which one the actual WaterSip accuracy in terms of estimating precipitation?

> Page 6, Line 18: In the sentence, "the two-month anomalies" is mentioned without giving the referring mean. By reading the caption of Figure 3 , I learn that this is the anomaly against the whole wet season (April-September). However, it is confusing without an explicit mention in the text.

> Section 3.5: I cannot find information on how is the local fraction of continental recycling calculated (and in Figure 8b). Without this information, I cannon justify the validity of the second-order continental moisture source, and therefore, the whole section 3.5. In my opinion, the innovation of this study largely comes from this section.

> Page 13, Lines 4-5: I do not know how is the mass-average moisture source distance defined. Is it defined from the furthest boundary of moisture source to the center of the YRV along the great circle? What does the deviation stand for, monthly variation or interannual variation? And, why there is not deviation for the centroid of moisture sources?

Printer-friendly version

Technical corrections:

Discussion paper

> Page 8, Lines 9-11: please put citations in the correct parentheses. 
> Page 9, Lines 24-25: this sentence is incomprehensible.

$>$ Figure 8. The red dashed lines is shown in the wrong panel; or the caption is wrong.

Interactive comment on Hydrol. Earth Syst. Sci. Discuss., https://doi.org/10.5194/hess-2018629, 2019.

Interactive

comment 